\title{
Letter to Mario Conte, Pete Cameron, and Chris Tschalaer regarding the Stern-Gerlach Force
}

W. MacKay

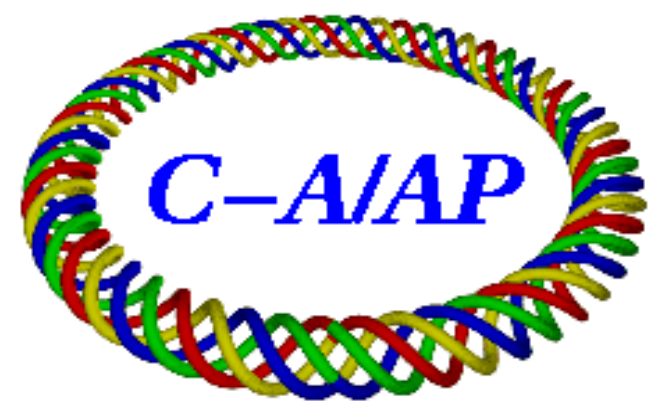

Collider-Accelerator Department Brookhaven National Laboratory

Upton, NY 11973 
23 July, 2006.

Dear Mario, Pete, and Chris,

Consider a rectangular traveling-wave structure with a TE mode having he following vector potential

$$
\vec{A}(x, y, z, t)=\left(\begin{array}{c}
\frac{B_{0}}{k} \sin \frac{\pi y}{b} \sin (k z-\omega t+\phi) \\
0 \\
0
\end{array}\right)
$$

where the beam moves parallel to the $z$-axis at height $y=b / 2$, and the phase velocity matches the initial particle velocity

$$
\beta c=v_{\mathrm{ph}}=\omega / k .
$$

The particle enters the structure at

$$
\begin{aligned}
& z_{0}=0 \\
& t_{0}=0
\end{aligned}
$$

and exits at

$$
\begin{aligned}
& z_{1}=L \\
& t_{1}=\frac{L}{\beta c}
\end{aligned}
$$

In the rest system of the particle these transform to

$$
\begin{aligned}
& z_{0}^{\diamond}=\gamma\left(z_{0}-\beta c t_{0}\right)=0 \\
& t_{0}^{\diamond}=\gamma\left(t_{0}-\beta z_{0} / c\right)=0
\end{aligned}
$$

and

$$
\begin{aligned}
& z_{1}^{\diamond}=\gamma\left(z_{1}-\beta c t_{1}\right)=0 \\
& t_{1}^{\diamond}=\gamma\left(t_{1}-\beta z_{1} / c\right)=\frac{L}{\gamma \beta c}
\end{aligned}
$$

The transverse coordinates are the same in both systems with $x^{\diamond}=x$ and $y^{\diamond}=y$.

Since there is no static potential, the vector potential transforms to

$$
\vec{A}^{\diamond}\left(x^{\diamond}, y^{\diamond}, z^{\diamond}, t^{\diamond}\right)=\left(\begin{array}{c}
A_{x}(x, y, z, t) \\
0 \\
0
\end{array}\right)
$$


however, this is still expressed with respect to the lab coordinates with

$$
A_{x}^{\diamond}\left(x^{\diamond}, y^{\diamond}, z^{\diamond}, t^{\diamond}\right)=\frac{B_{0}}{k} \sin \frac{\pi y}{b} \sin (k z-\omega t+\phi)
$$

Writing the coordinates in terms of the rest system this becomes

$$
\begin{aligned}
k z-\omega t & =k \gamma\left(z^{\diamond}+\beta c t^{\diamond}\right)-\omega \gamma\left(t^{\diamond}+\frac{\beta}{c} z^{\diamond}\right) \\
& =\gamma\left(k-\frac{\omega \beta}{c}\right) z^{\diamond}+\gamma(k \beta c-\omega) t^{\diamond} \\
& =\gamma k\left(1-\beta^{2}\right) z^{\diamond} \\
& =\frac{k}{\gamma} z^{\diamond}
\end{aligned}
$$

since we want the wave traveling with the particle, i. e., in the rest frame, the wave is stationary.

$$
\begin{aligned}
& \vec{E}^{\diamond}=-\frac{\partial \overrightarrow{A^{\diamond}}}{d t^{\diamond}}=0, \\
& \vec{B}^{\diamond}=\nabla^{\diamond} \times \vec{A}^{\diamond}=\left(\begin{array}{c}
\frac{B_{0}}{\gamma} \sin \frac{\pi y^{\diamond}}{b} \cos \left(\frac{k z^{\diamond}}{\gamma}+\phi\right) \\
-\frac{\pi B_{0}}{k b} \cos \frac{\pi y^{\diamond}}{b} \sin \left(\frac{k z^{\diamond}}{\gamma}+\phi\right)
\end{array}\right) .
\end{aligned}
$$

For $\vec{\mu}^{\diamond}=\mu^{\diamond} \hat{y}$

$$
\begin{aligned}
\vec{F}^{\diamond}\left(z^{\diamond}=0\right) & =-\nabla^{\diamond}\left(\vec{\mu}^{\diamond} \cdot \vec{B}^{\diamond}\right)=-\mu^{\diamond} \frac{\partial B_{y}^{\diamond}}{\partial z^{\diamond}} \\
& =\frac{\pi \mu^{\diamond} B_{0} k}{\gamma^{2}} \cos \phi
\end{aligned}
$$

and the final momentum in the rest system after the wave-guide passes by the particle is

$$
\begin{aligned}
p_{z 1}^{\diamond} & =\int_{t_{0}^{\diamond}}^{t_{1}^{\diamond}} F_{z}^{\diamond} d t^{\diamond} \\
& \simeq F_{z}^{\diamond} \frac{L}{\gamma \beta c} \\
& =\frac{\pi \mu^{\diamond} B_{0} k L}{\gamma^{3} \beta c} \cos \phi
\end{aligned}
$$

Not that the maximum kick comes from a phase of $\phi=0$ The final energy in the rest frame is approximately

$$
U_{1}^{\diamond}=\sqrt{m^{2} c^{4}+\left(p_{z 1}^{\diamond} c\right)^{2}}
$$


and boosting back to the lab this yields

$$
\begin{aligned}
U_{1} & =\gamma\left(\sqrt{m^{2} c^{4}+\left(p_{z 1}^{\diamond} c\right)^{2}}+\beta p_{z 1}^{\diamond} c\right) \\
& \simeq \gamma m c^{2}+\frac{\pi \mu^{\diamond} B_{0} k L}{\gamma^{2}} \cos \phi .
\end{aligned}
$$

So the energy change for this wave is only proportional to $1 / \gamma^{2}$.

Well this looks bad for a wave traveling with the particle. Naively one might expect that the wave length $2 \pi / k$ should have transformed as with $1 / \gamma$ since the waveguide must

be shortened by a factor of $1 / \gamma$ when boosted to the rest system. But the wave is moving with respect to the waveguide and is actually boosted to rest with a lengthened wavelength

$$
\lambda^{\diamond}=\gamma \lambda
$$

Comparing this with Compton scattering, we know that a photon traveling with the electron will barely change the electron's energy when it scatters. The large energy momentum transfers come from a head-on collision, so I guess we should expect this result.

In Eq. 50 of Ref 1., our problem is that we had the particle enter the cavity at a peak of the vertical magnetic field and exit a peak of the magnetic field of the opposite sign in order to get a significant energy shift $\left(\propto \gamma^{2}\right)$. This ignored the fact that a little bit farther upstream of the entrance and downstream of the exit, the particle must be in a field free region. In order to travel between the field free regions outside the cavity, into the peak fields at the edges of the cavity, the particle must experience gradients which are opposite to the gradient experienced inside the cavity. In effect the particle will experience a net energy change proportional to something like

$$
\int_{0-\epsilon}^{L+\epsilon} \frac{d f(z)}{d z} d z=f(L+\epsilon)-f(0-\epsilon)=0-0=0
$$

even though $f(0) \neq 0$ and $f(L) \neq 0$. Well outside the cavity the function $f(z)$ must be zero on both sides.

Presumably in Compton scattering the high momentum transfer for backscattering would have to come from the radiating of the transversely oscillating electron which would be driven by the rapidly oscillating $E_{x}^{\diamond}$ of the backwards traveling wave in the rest system.

Best ragards (standing on the chiappa de luogo),

Waldo

\section{Reference}

1. M Conte et al., INFN/TC-00/03, "The Stern Gerlach Interaction Between a Traveling Particle and a Time Varying Magnetic Field" (2000). 